How much higher can $T_{\mathrm{C}}$ go in $\mathrm{C}_{60}$ ? Schön and colleagues suggest that a further $1 \%$ increase in $\mathrm{C}_{60}$ separation might result in a $T_{\mathrm{C}}$ of around $150 \mathrm{~K}$. (The existing all-time record for superconductivity is $164 \mathrm{~K}$ for copper oxides subjected to extreme pressures.) Any greater expansion of $\mathrm{C}_{60}$ would probably destroy its intermolecular bonding completely, but there might be other, more intriguing ways to take advantage of the unique crystal structure of this compound. The separation between the electronic states and vibrational modes of $\mathrm{C}_{60}$ exploited by Schön et al. is just the sort of thing Little had in mind in the 1960s when he proposed his 'excitonic' model of superconductivity ${ }^{10}$. According to this idea, superconducting paired carriers are glued together by excitons with characteristic energies, unlike the several hundred degrees of phonons, of around $20,000 \mathrm{~K}$ - potentially yielding
$T_{\mathrm{C}}$ values well above room temperature. Could new excitonic states be introduced by clever chemical or structural modification of $\mathrm{C}_{60}$ molecules? Don't step off the elevator just yet.

Paul Grant is at the Electric Power Research

Institute, 3412 Hillview Avenue, Palo Alto,

California 94303, USA.

e-mail:pgrant@epri.com

1. Schön, J. H., Kloc, Ch. \& Batlogg, B. Science, 30 August 2001 (10.1126/science.1064773).

2. Jérome, D. et al. J. Phys. Lett. (Paris) 41, L95 (1980).

. Hebard, E. F. et al. Nature 350, 600-601 (1991).

4. Schön, J. H., Kloc, Ch. \& Batlogg, B. Nature 406, 702-704 (2000).

5. Schön, J. H., Kloc, Ch. \& Batlogg, B. Nature 408, 549-552 (2000)

6. Schön, J. H. et al. Nature 410, 189-192 (2001).

7. Schön, J. H., Kloc, Ch., Hwang, H. Y. \& Batlogg, B. Science 292, 252-254 (2001)

8. Bardeen, J., Cooper, L. N. \& Schrieffer, J. R. Phys. Rev. 108, 1175-1204 (1957)

9. Ramirez, A. P. et al. Phys. Rev. Lett. 68, 1058- 1060 (1992). 10. Little, W. A. Sci. Am. 212, 21 (1965).

\title{
Ecology
}

\section{Crowd trouble for predators}

\author{
Peter D. Moore
}

Flocking, herding, swarming: call it what you will. But when you're somebody's lunch there's safety in numbers, even when the predator is an aquatic plant.

A voiding being eaten is (obviously) one way that most organisms escape premature death. There are many techniques for evading predation, one of the most frequent being the herding or flocking

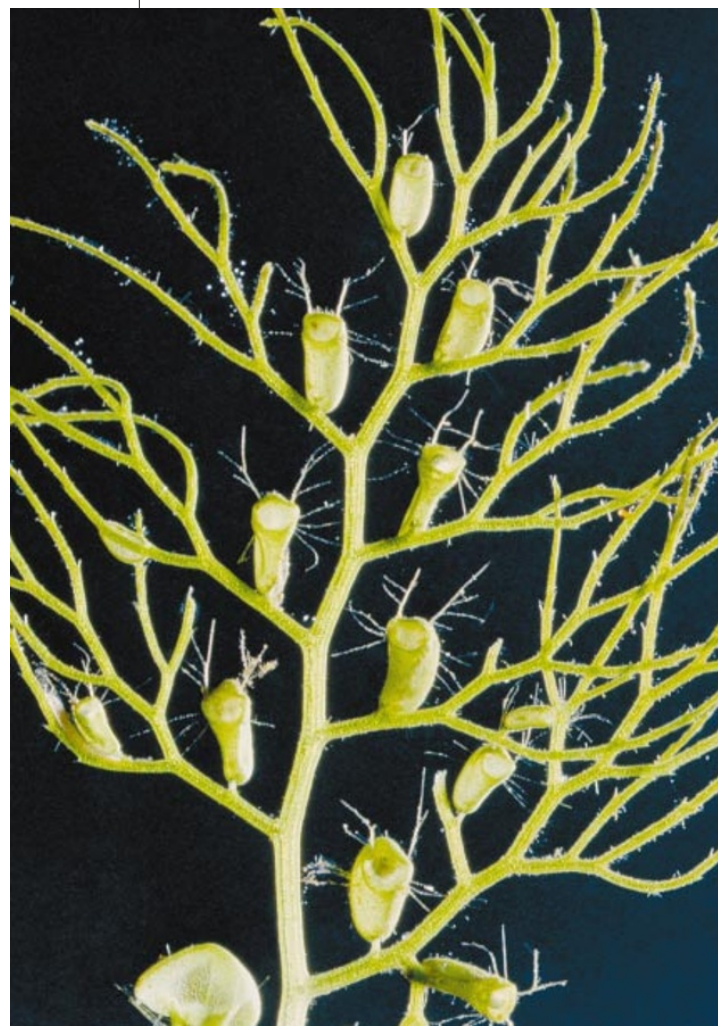

behaviour often seen in certain mammals, birds and fish. But what if the predator happens to be a plant? Does it still help to gather together in groups for mutual support? From work reported by Goran Englund and Sabine Harms (Oikos 94, 175-181; 2001), it seems that it does. Their finding in turn raises such questions as how the prey species (in this case, microscopic aquatic creatures called cladocerans) know when to swarm, and how the swarming mechanism operates.

The plant concerned, the bladderwort Utricularia vulgaris (Fig. 1), traps incautious zooplankton within its hollow leaves where it digests them and absorbs their nutrients. Although it is a sedentary predator, bladderwort has an active trapping mechanism, so its carnivory is not entirely passive. Sensory hairs on the outside of the bladder-like leaf detect the local presence of potential prey, resulting in the opening of a trap door and a rush of water and prey into the hollow leaf as negative pressure is released. But being static, the plant must rely on the errant prey organism colliding with the trap. One might predict, therefore, that the higher the density of the zooplankton, the more likely such collisions would become and the more successful the predator would be. Englund and Harms, however, show that at high densities

Figure 1 Bladderwort - armed and dangerous (at least to cladocerans). of cladoceran the number of prey trapped per bladder actually declines.

Observations of the behaviour of the prey, Polyphemus pediculus, revealed that they are either randomly or uniformly dispersed at low densities (20 individuals in a $125-\mathrm{ml}$ vessel), whereas at high densities (120 individuals per vessel) they aggregate into swarms. What constitutes the immediate stimulus for this response (such as visual or chemical signals) is unclear, but the flocking behaviour may well be the factor responsible for the reduced success of the predator. Two main changes occur in the swimming behaviour of individual cladocerans when they occur in a swarm they swim more slowly, and they follow a more contorted path than when they are dispersed.

Obviously, a slower-moving animal is less likely to encounter a static object within a given time, so the chances of a cladoceran hitting a trap are reduced if it slows down. It is also possible that at slower speeds the animal is better able to identify and avoid the traps. Again, it could be argued that a linear motion on the part of the prey is more likely to lead it to traps than random motion. However, given the sensory capacity of the Polyphemus, motion may not actually be random, but selective to avoid potentially dangerous objects, such as Utricularia leaves.

Another question that requires investigation is the possibility of predator satiation. If the plant has simply had its fill when its prey is abundant, then further increases in prey density would not increase trap success. But in these experiments trap success actually declines as density increases (over a threshold of about 35 animals per $125-\mathrm{ml}$ vessel). Also, the 'handling rate' for a trap the time it takes for the trapping mechanism to be reset - is around 10-15 minutes, so satiation is not a likely explanation of the observations.

It is of course possible that the swarming behaviour of Polyphemus is primarily the outcome of some entirely different behavioural demand. Perhaps it provides better feeding opportunities, or is related to reproductive cycles, or results in more efficient escape from mobile predators. Whatever the reason, swarming still gives the animal a better chance of avoiding consumption by a predatory plant.

Sticking together has proved an effective defence mechanism for many animals subjected to predation, from wildebeest to mackerel. The fact that it works even among microscopic creatures threatened by enemies as passive as a plant may provide insights into the benefits of the swarm.

Peter D. Moore is in the Division of Life Sciences,

King's College, Franklin-Wilkins Building, 150

Stamford Street, London SE1 9NN, UK.

e-mail:peter.moore@kcl.ac.uk 